\title{
ARTICLE
}

\section{New approach for describing nuclear reactions based on intra-nuclear cascade coupled with DWBA}

\author{
Shintaro Hashimoto ${ }^{a^{*}}$, Yosuke Iwamoto ${ }^{\mathrm{a}}$, Tatsuhiko Sato ${ }^{\mathrm{a}}$, Koji Niita ${ }^{\mathrm{b}}$, Alain Boudard ${ }^{\mathrm{c}}$, Joseph Cugnon ${ }^{\mathrm{d}}$, \\ Jean-Christophe David ${ }^{\mathrm{c}}$, Sylvie Leray ${ }^{\mathrm{c}}$ and Davide Mancusi ${ }^{\mathrm{c}}$ \\ ${ }^{a}$ Japan Atomic Energy Agency, 2-4 Shirakata, Tokai-mura, Naka-gun, Ibaraki-ken, 319-1195, Japan; ${ }^{b}$ Research Organization for \\ Information Science and Technology, 2-4 Shirakata, Tokai-mura, Naka-gun, Ibaraki-ken, 319-1106, Japan; ${ }^{c}$ CEA, Centre de \\ Saclay, IRFU/Service de Physique Nucléaire, F-91191 Gif-sur-Yvette, France; ${ }^{d}$ Fundamental Interactions in Physics and \\ Astrophysics, University of Liège, allée du 6 août 17, bât. B5, B-4000 Liège 1, Belgium
}

\begin{abstract}
We proposed a new nuclear reaction model in combination of Intra-Nuclear Cascade of Liège (INCL) and the Distorted Wave Born Approximation (DWBA) calculation, and incorporated it into the Particle and Heavy Ion Transport code System (PHITS). This model was applied to deuteron induced reactions on ${ }^{\text {nat }} \mathrm{Li}$ and ${ }^{9} \mathrm{Be}$ targets for deuteron incident energies of 40 and $18 \mathrm{MeV}$, respectively. Double-differential cross sections and neutron yields on thick targets obtained by PHITS using the model reproduce experimental data quite well.
\end{abstract}

\section{Keywords: Monte Carlo simulation; PHITS; nuclear reaction model; intra-nuclear cascade model; DWBA}

\section{Introduction}

Recently, accelerator-based neutron sources are utilized for scientific and medical applications, such as the International Fusion Materials Irradiation Facility (IFMIF) project and Boron Neutron Capture Therapy (BNCT). The $(d, x n)$ reactions for incident deuteron energies of $10-100 \mathrm{MeV}$ on targets of $\mathrm{Li}$, Be, and $\mathrm{C}$ play an important role in producing high intensity neutron sources.

Particle transport simulation based on Monte Carlo technique is very useful to predict particle fluxes or dose distribution in radiation facilities. We have developed the Particle and Heavy Ion Transport code System (PHITS) [1], which has been successfully applied to accelerator design, radiation therapy, cosmic-ray science, and so on.

However, Quantum Molecular Dynamics (QMD), which is a nuclear reaction model in PHITS for light-ion induced reactions, cannot reproduce experimental data of double-differential cross section (DDX) for the $(d, x n)$ reaction, and then also neutron yields for thick targets. Therefore, radiation transport calculations using PHITS for the above applications cannot be performed unless using special prescriptions, e.g. setting experimental data of DDX directly as neutron sources from the deuteron induced reaction. Those prescriptions can only be applied to the energy region where experimental data are available. Thus, a new reaction model reproducing DDX data is required.

\footnotetext{
*Corresponding author. Email: hashimoto.shintaro@jaea.go.jp
}

In this study, we propose a model that combines the Intra-Nuclear Cascade of Liège (INCL) [2] and the Distorted Wave Born Approximation (DWBA) models. The proposed model was verified with experimental data for two $(d, x n)$ reactions on ${ }^{\text {nat }} \mathrm{Li}$ and $\mathrm{Be}$ targets for incidences of 40 and $18 \mathrm{MeV}$ deuterons.

\section{Nuclear reaction model}

In the new model, INCL version 4.5 is employed as the fundamental model to describe the major part of nuclear processes. INCL can treat not only nucleons but also light ions $\left(d, t,{ }^{3} \mathrm{He}\right.$, and $\alpha$ ) as incident particles, and can be applied to collisions in the wide energy and target nucleus regions. Deuteron breakup and proton-stripping processes, which contribute to a broad peak in the $(d, x n)$ energy spectrum, are taken into account for INCL.

However, some discrete peaks in the high energy region of the spectrum cannot be described by INCL, since these peaks correspond to transitions between nuclear states based on the shell structure, which is not included in the INCL calculation. DWBA is a reaction model to obtain such cross sections on the basis of the quantum mechanical calculation. In DWBA, the nuclear reaction dynamics is described using wave functions, and the shell structure of nuclei is taken into account. DWBA calculation can give angular dependence of cross sections with interference pattern due to the quantum mechanical effect. Combing INCL with DWBA, we can obtain a nuclear reaction model taking account of important processes, which are neglected in 
QMD.

When a nuclear reaction event occurs in the PHITS calculation with the combination model, the generation of the DWBA event is randomly determined first, according to the ratio of an angular integrated cross section calculated by DWBA to the total reaction cross section. The angular differential and integrated cross sections are prepared as data tables in advance. If DWBA is chosen, results of one nuclear reaction event, which describes energies and angles of emitted neutron and residual nucleus, are randomly determined according to values of the data tables of the angular differential cross section. If DWBA is not chosen, INCL calculation is performed with the reduced maximum value of the impact parameter, $b_{\max }$ given by,

$$
b_{\max }=\sqrt{R^{2}-\sigma_{\mathrm{DWBA}} / \pi},
$$

where $R$ is an interaction radius corresponding to the total reaction cross section, and $\sigma_{\mathrm{DWBA}}$ is the integrated cross section of DWBA. The transition processes calculated by DWBA correspond to the so-called 'direct process', which takes place at the surface of the target nucleus. Therefore, the impact parameter for INCL should be reduced. Finally, in this case, results of one nuclear reaction event are given by INCL calculation.

In this study, we use the TWOFNR code [3] to make the data tables of the cross sections by DWBA. We used an optical potential between $d$ and the target parameterized by Han et al. [4] and that between $n$ and the residual nucleus by Watson et al. [5]. We adjusted two parameters of the optical potential for $d$ to reproduce experimental data. Residual nuclei of ${ }^{7,8} \mathrm{Be}$ and ${ }^{10} \mathrm{~B}$ are considered in the $(d, n)$ reactions on ${ }^{6,7} \mathrm{Li}$ and ${ }^{9}$ Be targets, respectively.

\section{Results and discussion}

Figure 1 shows angular dependence of cross sections of the ${ }^{6} \mathrm{Li}(d, n)^{7} \mathrm{Be}$ reaction for $15 \mathrm{MeV}$ deuterons. The result of DWBA denoted by the green line is a sum of the two contributions denoted by the red and blue lines, and adequately reproduces the experimental data [6] since the normalization factor of DWBA is adjusted.

For the ${ }^{9} \mathrm{Be}(d, n){ }^{10} \mathrm{~B}$ reaction at $7 \mathrm{MeV}$, angular differential cross sections are shown in Figure 2. Adjusting optical potential parameters, the red line reproduces the experimental data $[7,8]$ also in this case.

Figures 3(a) and 3(b) show double differential cross sections (DDXs) of the ${ }^{\text {nat }} \operatorname{Li}(d, x n)$ reaction for the incident energy of $40 \mathrm{MeV}$ at neutron emission angles of $0^{\circ}$ and $15^{\circ}$, respectively, calculated by PHITS. Experimental data [9] are compared with results of the PHITS calculation by three nuclear reaction models, QMD, INCL only, and the new model. The QMD results have large deviation from the experimental data. On the other hand, the results of INCL can reproduce broad peaks around $20 \mathrm{MeV}$ of experimental data due to the proton-stripping process, although there is a slight difference between the results and the data. Since INCL has been developed to consider reactions at incident energies of rather higher than $40 \mathrm{MeV}$, the internal parameters related with the process are not probably suitable for the low energy reaction. It will be improved by a modification of the parameters. Furthermore, the contributions by the DWBA calculation generate two peaks in higher neutron energy region from $50 \mathrm{MeV}$ to $60 \mathrm{MeV}$, and the new model describes the DDXs quite well. Although the new model does not reproduce only peaks around $40 \mathrm{MeV}$, this underestimation may be improved by taking more suitable parameters in the DWBA calculation. For the purpose, more experimental data of the ${ }^{6} \mathrm{Li}(d, n)^{7} \mathrm{Be}$ reaction are required.

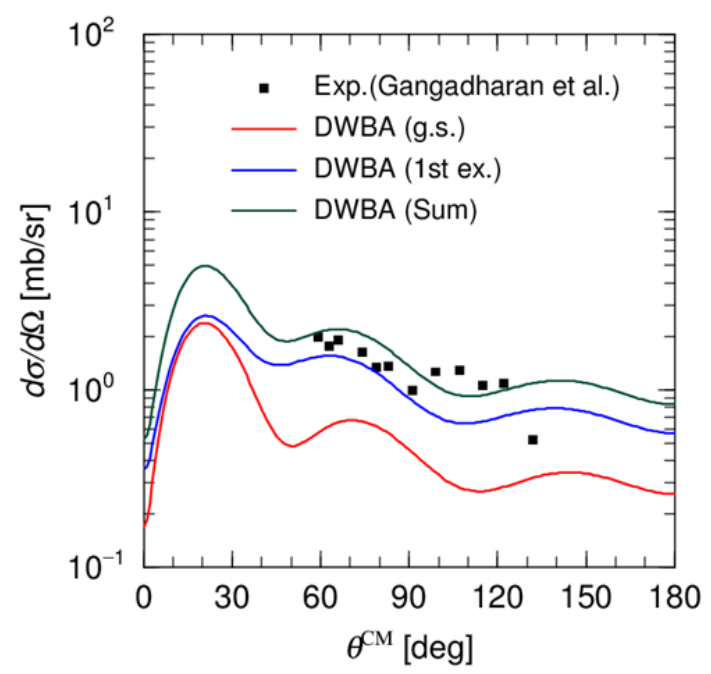

Figure 1. Angular differential cross sections of the ${ }^{6} \mathrm{Li}(d, n)^{7} \mathrm{Be}$ reaction for $15 \mathrm{MeV}$. Red and blue lines represent results of DWBA calculation for the transition to the ground and $1^{\text {st }}$ excited states of ${ }^{7} \mathrm{Be}$, respectively. Green line corresponds to a sum of them. Experimental data [6] correspond to such a sum.

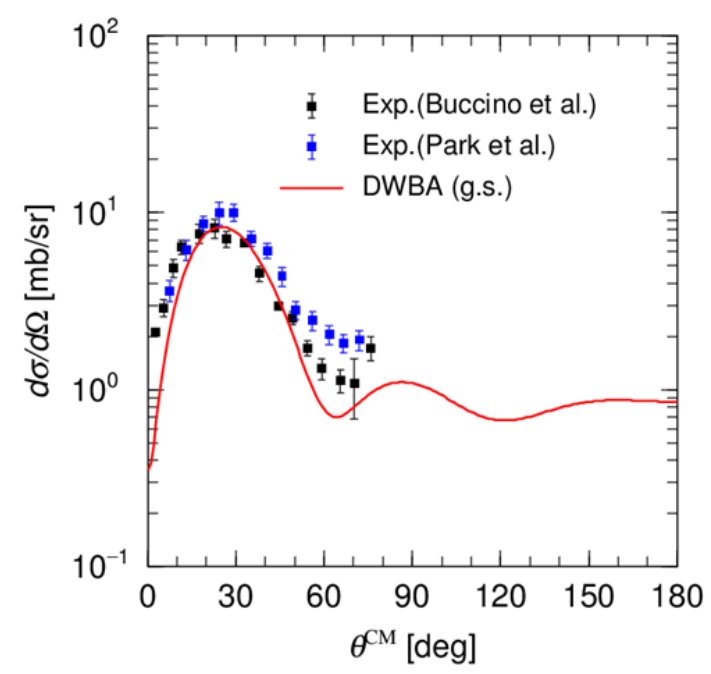

Figure 2. Angular differential cross sections of the ${ }^{9} \mathrm{Be}(d, n){ }^{10} \mathrm{~B}$ reaction for $7 \mathrm{MeV}$. Red line is a DWBA result corresponding to the transition to the ground state of ${ }^{10} \mathrm{~B}$. Black and blue boxes are experimental data $[7,8]$. 


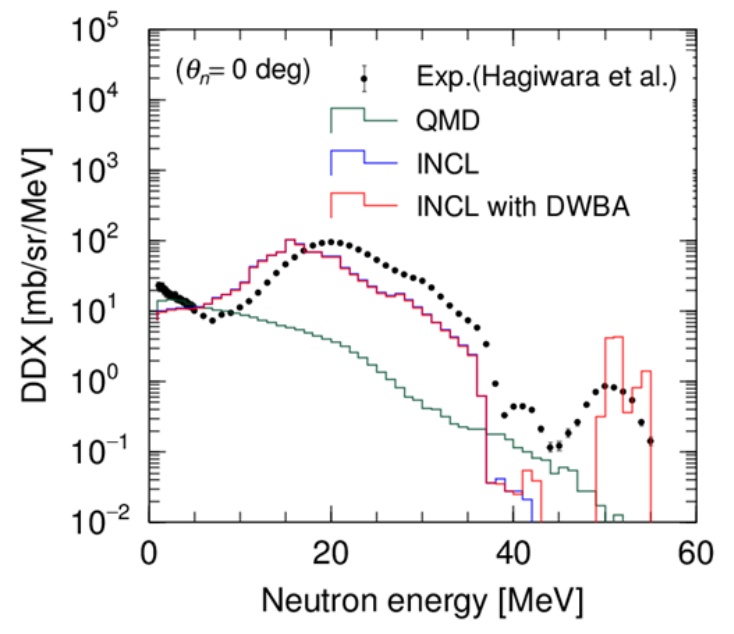

Figure 3(a). Double differential cross sections of the ${ }^{\text {nat }} \mathrm{Li}(d, x n)$ reaction at $40 \mathrm{MeV}$ at $0^{\circ}$ of the emission angle. Symbols represent experimental data [9]. Green, blue, and red lines correspond to results of the PHITS calculation using QMD, INCL only, and the new model, respectively. The blue and red lines agree with each other below $40 \mathrm{MeV}$.

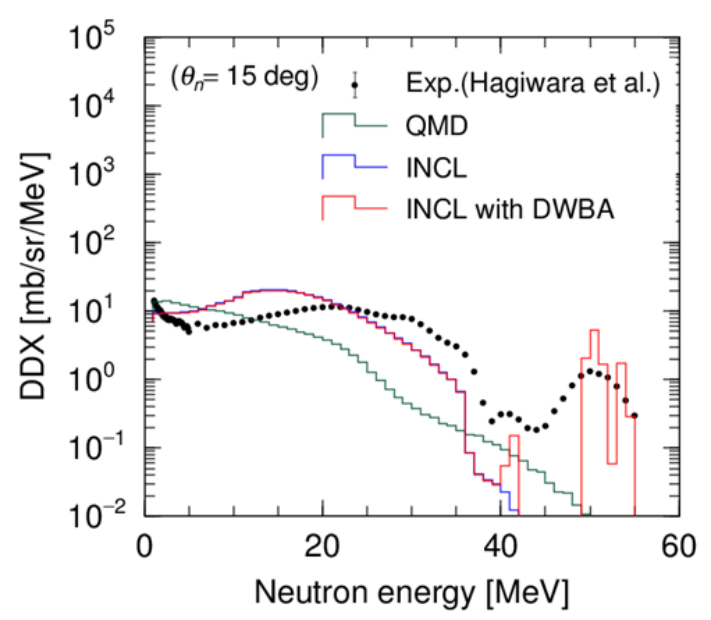

Figure 3(b). Double differential cross sections of the ${ }^{\text {nat }} \mathrm{Li}(d, x n)$ reaction at $40 \mathrm{MeV}$ at $15^{\circ}$ of the emission angle.

Figure 4(a) and 4(b) show neutron yields of the deuteron induced reaction on the $2.1 \mathrm{~cm}$ thick ${ }^{\text {nat }} \mathrm{Li}$ target at the $40 \mathrm{MeV}$ at the emission angles of $0^{\circ}$ and $15^{\circ}$, respectively. We compared results obtained by the three reaction models with experimental data [9]. Contribution of INCL and DWBA is the same as in the DDX results. The result of the PHITS calculation using the new model reproduces the experimental data quite well in the whole neutron energy region at both the forward emission angles unlike the DDX case.

For the ${ }^{9} \mathrm{Be}(d, x n)$ reaction at $18 \mathrm{MeV}, \mathrm{DDX}$ at the emission angle of $3.5^{\circ}$ is shown in Figure 5 . Experimental data [8] are reproduced by the result using the new model quite well. The agreement between the calculated result and the experimental data for the ${ }^{9} \mathrm{Be}$ target is better than that for the ${ }^{\text {nat }} \mathrm{Li}$ target. The reason is that we used the DWBA cross sections reproducing experimental data around $3.5^{\circ}$ for ${ }^{9} \mathrm{Be}(d, n){ }^{10} \mathrm{~B}$, as shown in Fig. 2. On the other hand, since experimental data at $0^{\circ}$ and $15^{\circ}$ for ${ }^{6,7} \operatorname{Li}(d, n)^{7,8} \mathrm{Be}$ are not available, the
DWBA results at the angles have an uncertainty in the ${ }^{\text {nat }} \mathrm{Li}$ target case.

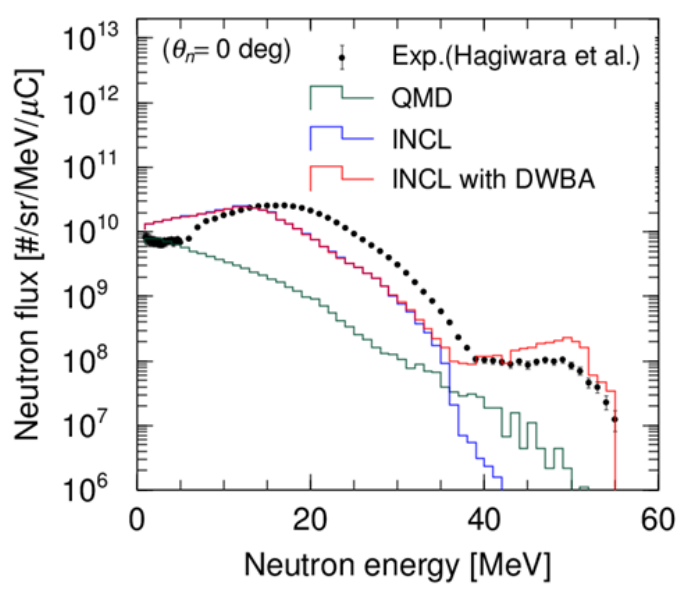

Figure 4(a). Neutron yields of the $(d, x n)$ reaction at $40 \mathrm{MeV}$ on the thick ${ }^{\text {nat }} \mathrm{Li}$ target at $0^{\circ}$.

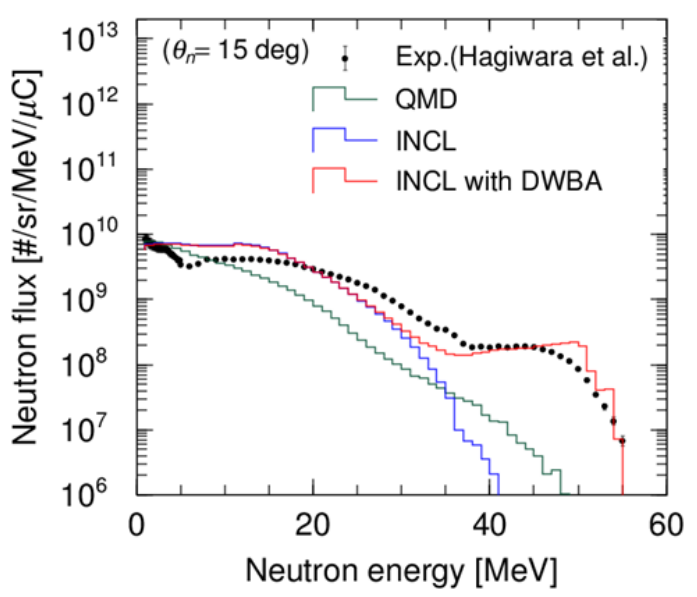

Figure 4(b). Neutron yields of the $(d, x n)$ reaction at $40 \mathrm{MeV}$ on the thick ${ }^{\text {nat }} \mathrm{Li}$ target at $15^{\circ}$.

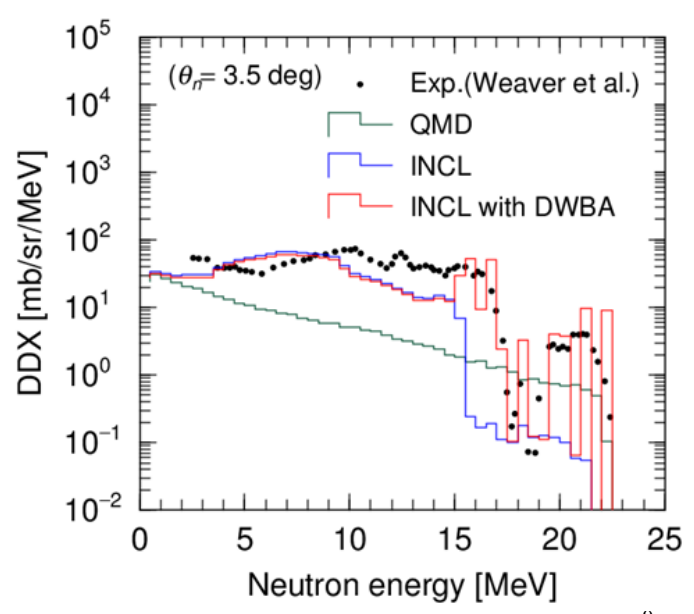

Figure 5. Double differential cross sections of the ${ }^{9} \mathrm{Be}(d, x n)$ reaction for $18 \mathrm{MeV}$ at $3.5^{\circ}$ of the emission angle. Green, blue, and red lines represent results of the PHITS calculation with QMD, INCL only, and the new model, respectively. Experimental data denoted by closed circles are taken from Ref. [10]. The blue and red lines agree with each other below $15 \mathrm{MeV}$. 
Figure 6 shows neutron yields of the $(d, x n)$ reaction on the $2.5 \mathrm{~mm}$ thick ${ }^{9} \mathrm{Be}$ target at the emission angle of $3.5^{\circ}$. In this case also, the result of the PHITS calculation (the red line) describes experimental data [10] by INCL and DWBA.

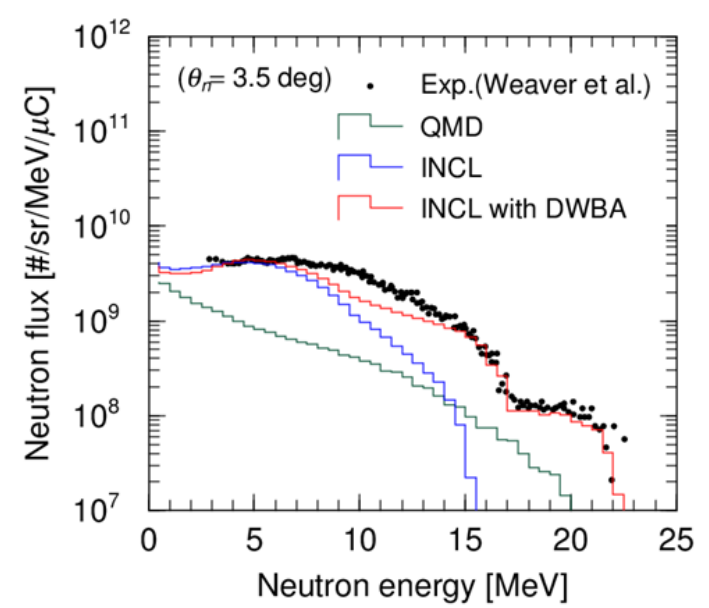

Figure 6. Neutron yields of the $(d, x n)$ reaction at $18 \mathrm{MeV}$ on the thick ${ }^{9} \mathrm{Be}$ target at $3.5^{\circ}$.

\section{Conclusion}

We proposed the new nuclear reaction model, where INCL describes the major part of the reaction processes and DWBA gives the peaks which correspond to transitions between the nuclear states. The model was applied to the $(d, x n)$ reactions for deuteron incident energies of 40 and $18 \mathrm{MeV}$ on the ${ }^{\text {nat }} \mathrm{Li}$ and ${ }^{9} \mathrm{Be}$ targets, respectively. The results of the PHITS calculation with the nuclear reaction models, QMD, INCL only, and the new combination model of INCL and DWBA, were compared with the experimental data of DDXs and neutron yields on the thick targets. We confirmed the improved reaction model gives a good agreement between the DDXs obtained by the calculation and the experimental data: the broad peaks due to the deuteron breakup and proton stripping processes are described by INCL, and some discrete peaks in the high energy region of the neutron spectra are reproduced by the DWBA calculation. Because of the agreement in the DDXs, the neutron yields on the thick targets calculated by PHITS reproduce the experimental data quite well. Therefore, calculations of particle transport including deuteron induced reactions will be adequately performed by PHITS with the new model, even if any special prescriptions are not applied. There is the advantage of the model that the production of proton or alpha also occurs in the reaction, while neutron spectra are only described in the case of using the prescription.

\section{References}

[1] K. Niita, N. Matsuda, Y. Iwamoto, H. Iwase, T. Sato, H. Nakashima, Y. Sakamoto and L. Sihver, PHITS: Particle and Heavy Ion Transport Code System, Version 2.23, JAEA-Data/Code 2010-022, Japan Atomic Energy Agency, (2010). http://phits.jaea.go.jp/index.html.

[2] A. Boudard and J. Cugnon, Joint ICTP-IAEA Advanced Workshop on Model Codes for Spallation Reactions, INDC(NDS)-0530, IAEA, (2008), pp. 29-50.

http://www-nds.iaea.org/reports-new/indc-reports/i ndc-nds/indc-nds-0530.pdf.

[3] M. Igarashi, Two-step finite-range DWBA code, TWOFNR.

http://www.nscl.msu.edu/ brown/reaction-codes/t wofnr/twofnr.pdf.

[4] Y. Han, Y. Shi, and Q. Shen, Deuteron global optical model potential for energies up to $200 \mathrm{MeV}$, Phys. Rev. C74 (2006), pp. 044615-1-11.

[5] B.A. Watson, P.P. Singh and R.E. Segel, Optical-model analysis of nucleon scattering from 1p-shell nuclei between 10 and $50 \mathrm{MeV}$, Phys. Rev. 182 (1969), pp. 977-989.

[6] S. Gangadharan and R.L. Wolke, Recoil angular distributions of the ${ }^{6} \mathrm{Li}(d, n){ }^{7} \mathrm{Be}$ and ${ }^{12} \mathrm{C}(d, n)^{13} \mathrm{~N}$ reactions, Phys. Rev. C1 (1970), pp. 1333-1341.

[7] S.G. Buccino and A.B. Smith, Levels in ${ }^{10} \mathrm{~B}$ excited by the ${ }^{9} \mathrm{Be}(d, n)$ reaction, Phys. Lett. 19 (1965), pp. 234-237.

[8] Y.S. Park, A. Niiler and R.A. Lindgren, Spectroscopy of ${ }^{10} \mathrm{~B}$ levels from the ${ }^{9} \mathrm{Be}(d, n){ }^{10} \mathrm{~B}$ reaction, Phys. Rev. C8 (1973), pp. 1557-1573.

[9] M. Hagiwara, T. Itoga, N. Kawata, N. Hirabayashi, T. Oishi, T. Yamauchi, M. Baba, M. Sugimoto and T. Muroga, Measurement of neutron emission spectra in $\operatorname{Li}(d, x n)$ reaction with thick and thin targets for 40-MeV deuterons, Fusion Sci. Technol. 48 (2005), pp. 1320-1328.

[10]A.K. Weaver, J.D. Anderson, H.H. Barschall and J.C. Davis, Neutrons from the bombardment of beryllium by deuterons, Phys. Med. Biol. 18 (1973), pp. 64-70. 